\title{
A Brief Introductory Review of Information Thermodynamics
}

\author{
Tahara Hiroki
}

May 21, 2020

\begin{abstract}
The aim of this review is to introduce the research fields that integrate information theory and thermodynamics referred to as information thermodynamics the most briefly.
\end{abstract}

\section{Introduction}

The origin of the research fields that integrate information theory and thermodynamics can be traced back to Maxwell's thought experiment "Maxwell's demon"[1]. This concept can be formulated as reducing total entropy in the system by feedback control based on measurements at the level of thermal fluctuations[2][3], and this seems to contradict the second law of thermodynamics $[4][2][3]$.

Theoretical discussions on this issue have advanced rapidly in the last decade or so[2], and specifically it has been revealed that incorporating the concept of information[5] [6] into research results in non-equilibrium statistical mechanics[7] [8][9] allows a completely exact understanding of the consistency between "demon" and the second law of thermodynamics[2][5].

In addition to this, experimental advances in research on the "demon" have recently begun[2]. Specifically, "demon" was realized by experiment actually[10] due to the advances of experimental technologies to measure mycro thermodynamical systems and control them by feedback[2][3][10].

In this way, researches that integrate information theory and thermodynamics have been forming new research fields, and it can be referred to as information thermodynamics[5][11][12]. The studies on information thermodynamics not only solve the issue of "Maxwell's demon" but olso reveal more abundant discoveries[2]. For instance, it became revealed that the upper limit of the amount of work that the "demon" can retrieve and the theoretical lower limit of the energy cost required for measurement are quantitatively linked to the 'amount of information'[12]. The aim of this review is to introduce information thermodynamics the most briefly. This review is organized as follows:後で付け 足す We only consider classical systems[13]. 


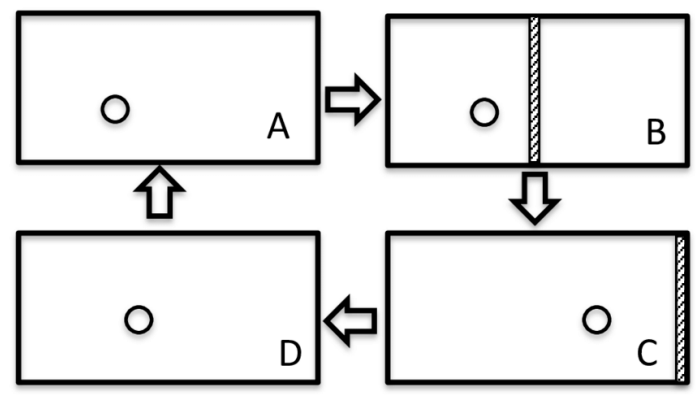

Figure 1:

$\lceil$ Schematic of the original Szilard engine. (A) There is a single molecule inside the box. (B) Inserting a piston divides the box into two halves with the molecule trapped on one side of the piston. (C) The pressure from the single molecule pushes the piston to one end of the box performing work. (D) Finally, the piston is removed and the system completes its cycle.」

(Quo, 2014)[16]

\section{Szilard Engine}

Let us start from a simple thought experiment, "Szilard engine" [14], the singleparticle heat engine model known as the simplest and the most essential model of "Maxwell's demon" [15]. (See Figure 1) First, as shown by (A) in Figure 1, one particle is in a box that is in contact with a heat bath[17][18] at temperature $T$, whose system is in thermal equilibrium[19]. Second, as in (B), we put a partition in the center of the box. Given that the particle is small enough, no work is required for this operation[20]. Then, the observer "demon" measures which box the particle is in without error. According to the definition described later, the information content[21], one of the kinds of the 'amount of information'[22] obtained by the measurement is $1[\mathrm{bit}]=\ln 2$. After the measurement, "demon" is completely aware of which box the particle is in. Therefore, as shown in (C), "demon" can move the partition in the isothermal quasi-static process as follows: if the particle is in the left, the partition is moved to the right end, and if it is in the right, it is moved to the left end i.e. "demon" doubles the volume of the box containing the particle[2]. Due to ideal gas law[23][24]

$$
p V=n R T \Longleftrightarrow p V=\frac{N}{N_{\mathrm{A}}} R T \Longleftrightarrow p=\frac{R}{N_{\mathrm{A}}} \frac{N T}{V} \Longleftrightarrow p=k_{\mathrm{B}} \frac{N T}{V}
$$

$N=1$ and $F=p S[25]$, the work done by the particle is as follows:

$$
W=\int_{V_{0}}^{2 V_{0}} F \mathrm{~d} x=\int_{V_{0}}^{2 V_{0}} k_{\mathrm{B}} \frac{T}{S x} S \mathrm{~d} x=k_{\mathrm{B}} T \int_{x_{0}}^{2 x_{0}} \frac{1}{x} \mathrm{~d} x=k_{\mathrm{B}} T \ln 2
$$

As shown in (D), removing the partition without giving work[20], the system returns to its original state in thermal equilibrium[19] shown by (A). Therefore we can get $k_{\mathrm{B}} T \ln 2$ work from this engine. 
At the model of Szilard engine, there is only one heat bath[17][18] and the first and last states are in the same thermal equilibrium[19] even we can get positive work from this. This seems to contradict the secound law of thermodynamics[5]: 「It is impossible to devise a cyclically operating device, the sole effect of which is to absorb energy in the form of heat from a single thermal reservoir and to deliver an equivalent amount of work.[26]」 (Kelvin-Planck statement[27]). This is the paradox of "Maxwell's demon" [2] [28]. Historically, it was difficult to solve this problem clearly from the viewpoints of thermodynamics[29] or statistical mechanics[30][2]. Although many physicists such as Brillouin[31], Landauer[32], and Bennett[33] have been discussing this issue[34] since Szilard advocated this thought experiment in 1929[14], each of these discussions had essential problems[2]. The paradox was completely solved in the 21st century, which required information theory established in the middle of the 20th century and knowledge of non-equilibrium statistical mechanics developed in the last 20 years[2][35].

For the discussion below, there are two most important points on this discussion of Szilard engine. One of them is that the essence of operation of "Maxwell's demon" is to perform feedback control[36][37] based on measurements at the level of thermal fluctuations[3]. Another important point is that the amount of work $k_{\mathrm{B}} T \ln 2$ extracted in the cycle is proportional to the 'amount of information' $\ln 2$ obtained in the measurement, with $k_{\mathrm{B}} T$ as the proportional constant. This means that the feedback is able to transform information into work[2] [3], as described later. A heat engine that converts information into work is referred to as an information heat engine[38].

\section{Quantities in Information Theory}

As mentioned above, concepts in infomation theory[6] especially the 'amount of information' [22] are important to develop the studies of information thermodynamics[2][5]. In this section, we define the 'amount of information' and a related quantity, mutual information[22][39].

\section{1 'Amount of Information'}

In information theory, the information content[40], the quantity of the information that event $A$ with probability $P(A)[41]$ has occurred which means the level of "surprise" [42] of $A$ is defined as follows[43]:

$$
I(A):=\log \frac{1}{P(A)}=-\log P(A)
$$

and the information entropy[40], the quantity of the unsureness of the information of the given discrete stochastic variable[44] (or any kinds of random element[45]) $X$ is defind as the expected value[46] of the information contents 
of the elementary events as follows[2][43]:

$$
S(X):=\mathrm{E}[I(X)]=\sum_{x \in X} P(x) I(x)=-\sum_{x \in X} P(x) \log P(x)
$$

Strictly speaking, $[x \in X]$ means $[x \in X(\Omega)][47]$ and $[P(x)]$ means $[P(\{x\})][48]$.

Here, the base of log is arbitrary. This is because even if the base changes, the value changes only by a constant factor, and essentially does not change[49]. The dimension of this quantity [50] is 1[51], however since the value differs depending on the base used for the definition, the unit may be defined for distinction, such as [bit] when the base is 2, [nat] when $e$ is and [dit] when 10 is[52] like [rad] and the arc degree[53]. In this review, we use $e$ as the base i.e. we define the quantities as follows:

$$
\begin{gathered}
I(A):=-\ln P(A) \\
S(X):=-\sum_{x \in X} P(x) \ln P(x)
\end{gathered}
$$

Let $N(<\infty)$ be the number of possible cases of $X$,

$$
0 \leq S(X) \leq \ln N
$$

$S(X)=\ln N$ is established when $P(x)=1 / N$ for any $x \in X$ i.e. the distribution is completely random[54]. On the other hand, $S(X)=0$ is established at a deterministic case[55], where $P(X)=1$ for only one $X$ i.e. there is no randomness.

At the case of the measurement at the model of Szilard engine[14] for instance, $X$ is which box the particle is in i.e. $R$ or $L$. Due to $N=|\{R, L\}|=2$ and they are equally possible[56],

$$
P(R)=P(L)=\frac{1}{2}
$$

Therefore the information entropy about the information of which the particle is in is as follows:

$$
S(X)=-\sum_{x \in\{L, R\}} P(x) \ln P(x)=-\left(\frac{1}{2} \ln 2^{-1}\right) \times 2=\ln 2
$$

and the information content about the gotten information is as follows:

$$
I(R)=I(L)=\ln 2
$$

The name 'information entropy' is simply because the form of the formula is similar to the entropy in statistical physics[57] i.e. the origin of information entropy has nothing to do with statistical mechanics[40]. However, it seems that the entropy in statistical mechanics and the entropy in information theory are almost the same in some cases[2]. If $X$ follows a microcanonical distribution[58] for instance, the information entropy $S(X)$ is expressed as follows:

$$
S(X)=\ln N
$$


as mentioned above, and this is almost exactly the same as Boltzmann equation[59]

$$
S=K_{\mathrm{B}} \ln W
$$

except for constant multiplication. These similarities suggest that information theory and thermodynamics are closely related[14].

\subsection{Mutual Information}

The quantity referred to as mutual information, which describes the degree of correlation between two random variables $X$ and $Y$ is defined as follows[60]:

$$
I(X ; Y):=S(X)+S(Y)-S(X, Y)=\sum_{(x, y) \in X \times Y} P(x, y) \ln \frac{P(x, y)}{P(x) P(y)}
$$

Strictly speaking, $[S(X, Y)]$ means $[S((X, Y))][61]$, and it is strictly defined because $(X, Y)$ is one of the random elements[45]. As can be seen from the definition, mutual information quantifies how much the two stochastic variables $X$ and $Y$ are correlated[2] i.e. how much information they share, depending on how much the entropies of the two variables are not additive[60].

Generally,

$$
0 \leq I(X ; Y) \leq \min \{S(X), S(Y)\}
$$

$\mathrm{I}(\mathrm{X} ; \mathrm{Y})=0$ is established when $\frac{P(x, y)}{P(x) P(y)}=0$ i.e. $X$ and $Y$ are stochastically independent [62][63][64]. On the other hand, $I(X ; Y)$ takes the maximum when $x=y$ for all $(x, y)$ i.e. $I(X ; Y)=S(X)=S(Y)$ i.e. $X$ and $Y$ are perfectly correlated[65]. This also seems intuitively correct.

\subsection{For Continuous Stochastic Variables}

For continuous stochastic variables[66], these two quantities which are dependent variables[67] of stochastic variables[44] (i.e. except information content) are defined as follows[68]:

$$
\begin{gathered}
S(X):=-\int_{x \in X} P(x) \ln P(x) \mathrm{d} x \\
I(X ; Y):=\int_{(x, y) \in X \times Y} P(x, y) \ln \frac{P(x, y)}{P(x) P(y)} \mathrm{d} x \mathrm{~d} y
\end{gathered}
$$

In the case of continuous variables, mutual information is invariant to variable transformation[2], but information entropy is not invariant. Moreover, the information entropy is not generally non-negative, and the inequality related to the upper limit of mutual information does not always hold. 


\section{Derivation of the Extended Second Law of Thermodynamics from Jarzynski Equality}

By the way, in order to consider thermodynamics and information theory collectively, not only the quantities in information theory but also the basic equations in thermodynamics must be taken as prerequisite knowledge[2][5]. Especially, the second law of thermodynamics is one of the keys of this discussion[4]. Therefore in this section, we introduce Jarzynski Equality[69], which is considered as more basic equation on the entropy enhancement[70] and derive the second law from this equation.

Now, we consider the thermodynamical system which receives work $W$ from the outside while in contact with a heat bath at temperature $T$ and evolves from time $t=0$ to $t=\tau$. In this process, the system absorbs heat $Q$ from the heat bath, and if the energy change of the system is $\Delta E$, the first law of thermodynamics[71]

$$
\Delta E=W+Q
$$

holds. If the variable that describes the micro state[72] of the system is $x$, since the system is affected by the heat bath and the heat bath is thermally fluctuating[73], the time evolution $x(t)$ is described as a stochastic process[74]. Since work $W$ and heat $Q$ depend on $x(t)[30], W$ and $Q$ are also stochastic variables.

If the probability density of $x$ at time $t$ is $P(x, t)$ [41], the information content of $x=x_{\lambda}$ can be defined as follows:

$$
I\left(x_{\lambda}, t\right):=-\ln P\left(x_{\lambda}, t\right)
$$

Note that the random variable is considered as continuous in consideration of the cases such as where the microscopic motion is described by the Langevin equation[75]

$$
m \frac{\mathrm{d} \boldsymbol{v}}{\mathrm{d} t}=-\beta \boldsymbol{v}+\boldsymbol{\eta}(t)
$$

however the same discussion can be applied to discrete systems[68]. Here, given one of $x(t)$, let the change of information content be

$$
\Delta I:=I(x, \tau)-I(x, 0)
$$

and the inverse temperature[29] be

$$
\beta:=\left(k_{\mathrm{B}} T\right)^{-1}
$$

stochastic entropy production in the whole system including the system and the heat bath can be defined as follows:

$$
\langle\sigma\rangle:=\Delta S-\beta\langle Q\rangle
$$

with

$$
\sigma:=\Delta I-\beta Q
$$


it being clear that $\Delta I$ and $\sigma$ are stochastic variables depending on $x(t)$ [73]. This is because $\Delta S$ and $-\beta Q$ can be considered as stochastic entropy changes of the system and the heat bath respectively[2][29].

At this situation, an equation holds for any $x(t)$ that can take any state including non-equilibrium state at any time as follows[70]:

$$
\left\langle e^{-\sigma}\right\rangle=1
$$

This is reffered to as Jarzynski equality[69]. Due to Jensen inequality[76]

$$
\int_{-\infty}^{\infty} f(y(x)) p(x) d x \geq f\left(\int_{-\infty}^{\infty} y(x) p(x) d x\right)
$$

for convex $f$ and exponential function is convex[77], an inequality holds as follows:

$$
\left\langle e^{-\sigma}\right\rangle \geq e^{-\langle\sigma\rangle}
$$

Therfore

$$
e^{-\langle\sigma\rangle} \leq 1
$$

i.e.

$$
\langle\sigma\rangle \geq 0
$$

i.e. the entropy production of the whole system it is always nonnegative on average even it can be negative stochastically. From the definition of $\sigma$, this can also be expressed as follows:

$$
\Delta S=\langle\Delta I\rangle \geq \beta\langle Q\rangle
$$

This is exactly the extension of the second law of thermodynamics to nonequilibrium states using information entropy as non-equilibrium entropy because this holds for any $x(t)$ including non-equilibrium states[70] and the second law for equilibrium states[26]

$$
-\langle W\rangle \leq 0
$$

can be derived by this[70].

In this way, Jarzynski equality contains the second law of thermodynamics and can be considered as a stronger representation of it in the form of an equation. In a macro thermodynamical system, the fluctuation of $\sigma$ is much smaller than the average value, and its effect is difficult to observe, however in a micro thermodynamical system such as Szilard engine, it is actually Jarzynski equality can be experimentally verified by observing the fluctuation of $\sigma[78][79]$.

\section{References}

[1] MAXWELL, J. Clerk. Theory of Heat. Astronomical register, 1872, 10: 107-107.

[2] SAGAWA, Takahiro. Thermodynamics of Information Processing. Bussei Kenkyu at Web, 2015, 4.1: 041209. 
[3] MATSUMOTO, Takumi; SAGAWA, Takahiro. Role of sufficient statistics in stochastic thermodynamics and its implication to sensory adaptation. Physical Review E, 2018, 97.4: 042103.

[4] SHIRAI, Kouun. Theory of the Scond Law of Thermodynamics. Osaka University, 2015, http://www.cmp.sanken.osaka-u.ac.jp/

[5] GRAY, Robert M. Entropy and information theory (1st ed., corrected). Springer Science \& Business Media, 1990.

[6] STONE, James V. Information Theory: A Tutorial Introduction. 2018.

[7] PRUESSNER, Gunnar. NON-EQUILIBRIUM STATISTICAL MECHANICS. Imperial College London, 2015.

[8] LE BELLAC, Michel. Non equilibrium statistical mechanics. 2007.

[9] CHOU, T.; MALLICK, K.; ZIA, R. K. P. Non-equilibrium statistical mechanics: from a paradigmatic model to biological transport. Reports on progress in physics, 2011, 74.11: 116601.

[10] TOYABE, Shoichi; et al. Experimental demonstration of information-toenergy conversion and validation of the generalized Jarzynski equality. Nature physics, 2010, 6.12: 988-992.

[11] SAGAWA, Takahiro; UEDA, Masahito. Information Thermodynamics: Maxwell's Demon in Nonequilibrium Dynamics. Nonequilibrium Statistical Physics of Small Systems: Fluctuation Relations and Beyond, 2013, 181-211.

[12] PARRONDO, Juan MR; HOROWITZ, Jordan M.; SAGAWA, Takahiro. Thermodynamics of information. Nature physics, 2015, 11.2: 131-139.

[13] SOMSIKOV, V. M. Thermodynamics and classical mechanics. Journal of physics: Conference series. IOP Publishing, 2005. p. 7.

[14] SZILARD, Leo. Über die Entropieverminderung in einem thermodynamischen System bei Eingriffen intelligenter Wesen. Zeitschrift für Physik, 1929, 53.11-12: 840-856.

[15] KIM, Sang Wook, et al. Quantum szilard engine. Physical review letters, 2011, 106.7: 070401.

[16] GUO, Quanmin. The Broken Engine of Szilard. arXiv:1410.8565, 2014.

[17] BRODY, Dorje C.; HUGHSTON, Lane P. Thermodynamics of quantum heat bath. Journal of Physics A: Mathematical and Theoretical, 2016, 49.42: 425302 .

[18] BRODY, Dorje C.; HUGHSTON, Lane P. Quantum Heat Bath. 2014. 
[19] GIBBONS, Richard Martin. Thermodynamic Equilibrium. Journal of Energy and Power Engineering, 2016, 10.10: 623-627.

[20] VAN ROON, P. H.; VAN SPRANG, H. F.; VERDONK, A. H. 'Work' and 'heat': On a road towards thermodynamics. International Journal of Science Education, 1994, 16.2: 131-144.

[21] CROCKER, Matthew W.; DEMBERG, Vera. Surprisal Theory and Empirical Evidence, 2015.

[22] NAKAHARA, Hiroyuki. The Amount of Information. Brain Science Dictionary, 2012.

[23] ARNAUD, Jacques; CHUSSEAU, Laurent; PHILIPPE, Fabrice. On the ideal gas law. Entropy, 2013, 15.3: 960-971.

[24] MENIN, Boris. The Boltzmann constant: Evaluation of measurement relative uncertainty using the information approach. Journal of Applied Mathematics and Physics, 2019, 7.3: 486-504.

[25] AHMADI, B., et al. Quantum Thermodynamic Force and Flow. arXiv:1802.09953, 2018.

[26] RAO, Y. V. C. Chemical engineering thermodynamics. Universities Press, 1997.

[27] SARASUA, L. G.; ABAL, G. Equivalence of the Kelvin-Planck statement of the second law and the principle of entropy increase. European Journal of Physics, 2016, 37.5: 055103.

[28] AYDIN, Alhun; SISMAN, Altug; KOSLOFF, Ronnie. Landauer' s Principle in a Quantum Szilard Engine without Maxwell 's Demon. Entropy, 2020, 22.3: 294.

[29] TAKAHASHI, Kazutaka. Thermodynamics. Tokyo Institute of Technology, 2018.

[30] TOMITA, Hiroyuki. Lecture Note of 'Statistical Physics'. (3rd ed.) Kyoto University, 2005.

[31] BRILLOUIN, Leon. Physical entropy and information. II. Journal of Applied Physics, 1951, 22.3: 338-343.

[32] LANDAUER, Rolf. Irreversibility and heat generation in the computing process. IBM journal of research and development, 1961, 5.3: 183-191.

[33] BENNETT, Charles H. The thermodynamics of computation-a review. International Journal of Theoretical Physics, 1982, 21.12: 905-940.

[34] LEFF, Harvey; REX, Andrew F. (ed.) Maxwell's Demon 2: Entropy, Classical and Quantum Information, Computing. CRC Press, 2002. 
[35] MÜLLER, Ingo. A history of thermodynamics: the doctrine of energy and entropy. Springer Science and Business Media, 2007.

[36] PHILLIPS, Charles L.; HABOR, Royce D. Feedback control systems. Simon and Schuster, Inc., 1995.

[37] DOYLE, John C.; FRANCIS, Bruce A.; TANNENBAUM, Allen R. Feedback control theory. Courier Corporation, 2013.

[38] TOYABE, Shoichi; et al. Information heat engine: converting information to energy by feedback control. arXiv:1009.5287, 2010.

[39] CHURCH, Kenneth Ward; HANKS, Patrick. Word association norms, mutual information, and lexicography. Computational linguistics, 1990, 16.1: $22-29$.

[40] SHANNON, Claude E. A mathematical theory of communication. Bell system technical journal, 1948, 27.3: 379-423.

[41] GRINSTEAD, Charles Miller; SNELL, James Laurie. Introduction to probability. American Mathematical Soc., 2012.

[42] MUKHERJEE, S. K. Self-Information and Information. Krishna Chandra College, 1976.

[43] KIDA, Takuya. Information Content and Entropy. Lecture 'Information theory', Hokkaido University, 2019.

[44] MAThAi A.M., RATHIE P.N. A Stochastic Variable. Probability and Statistics. Palgrave Macmillan, London, 1977.

[45] PROKHOROV, Yu.V. Random element. Encyclopedia of Mathematics, 1977.

[46] COMBLEY, Roz; et al. Expected Value. Cambridge business English dictionary, 2011.

[47] GORDON, Julia. Images of sets. Maths220 - Images and preimages, The University of British Columbia, 2020.

[48] OGAWA, Tomohiro. Probability Theory (beta version). The University of Electro-Communications, 2010.

[49] SCHNEIDER, Thomas D. Information theory primer with an appendix on logarithms. National Cancer Institute. 2007.

[50] HIROTA, Minoru. Dimension of Physical Quantity and Derived Units. Chemical Education, Chemical Society of Japan, 1986, 34.1:48-51.

[51] SHIROKOV, K. P. Dimensionless Quantities. The Great Soviet Encyclopedia (3rd ed.), 1970. 
[52] IMAI, Hideki. Theory of Information. (2nd ed.) 2019.

[53] ROBERTS, Donna. Arc Length and Radian Measure Math Bits Notebook, 2012.

[54] KINGMAN, John. Completely random measures. Pacific Journal of Mathematics, 1967, 21.1: 59-78.

[55] WALTER, Elizabeth; et al. Deterministic. Cambridge Advanced Learner's Dictionary and Thesaurus, 2005.

[56] DONOHUE, Kevin Dominic. Equally Likely Outcomes Approach. EE421. University of Kentucky, 1997.

[57] FEYNMAN, R. P. Feynman Lectures on Computation. Addis-on-Wesley, 1996.

[58] GROS, Claudius. Microcanonical Ensemble. Skript der 'Thermodynamik und Statistische Mechanik - Theoretische Physik V', Goethe-Universität Frankfurt am Main, 2017.

[59] SHARP, Kim. Entropy according to Boltzmann. University of Pennsylvania, 2016.

[60] LATHAM, Peter E.; ROUDI, Yasser. Mutual Information. Scholarpedia, 2009, 4(1):1658.

[61] GRISHIN, V.N. Tuple. Encyclopedia of Mathematics, 2016.

[62] COOK, John D. Stochastic Independence. 2008.

[63] MOESCHLIN, O.; et al. Stochastic Independence. Skript der '01196 Einführung in die Mathematische Stochastik', Die FernUniversität in Hagen, 2006.

[64] KLIBANOFF, Peter. Stochastically independent randomization and uncertainty aversion. Economic Theory, 2001, 18.3: 605-620.

[65] WALTER, Elizabeth; et al. Correlated. Cambridge Advanced Learner's Dictionary and Thesaurus, 2005.

[66] SIMON, Laura; et al. Lesson 14: Continuous Random Variables. STAT 414: Introduction to Probability Theory, Eberly College of Science, Pennsylvania State University, 2018.

[67] WALTER, Elizabeth; et al. Dependent Variable. Cambridge Advanced Learner's Dictionary and Thesaurus, 2005.

[68] OZAWA, Toru. Discrete and Continuous, Hokkaido University, 2007. 
[69] COHEN, E. G. D.; MAUZERALL, David. A note on the Jarzynski equality. Journal of Statistical Mechanics: Theory and Experiment, 2004, 2004.07: P07006.

[70] SAGAWA, Takahiro; UEDA, Masahito. Jarzynski Equality with Maxwell's Demon. RIMS Kôkyûroku, 2007, 1565: 98-106.

[71] BORYSOWICZ, Jerzy. The first law of thermodynamics. 2001.

[72] MCGOVERN, Judith Ann. Microstates and Macrostates. PHYS20352 Thermal and Statistical Physics, The University of Manchester, 2016.

[73] DE ZARATE, Jose M. Ortiz; SENGERS, Jan V. Fluctuations in fluids in thermodynamic equilibrium. Hydrodynamic fluctuations in fluids and fluid mixtures. Elsevier, 2006.

[74] ATZBERGER, Paul J. Stochastic Eulerian Lagrangian methods for fluid-structure interactions with thermal fluctuations. Journal of Computational Physics, 2011, 230.8: 2821-2837.

[75] SEKIMOTO, Ken. Langevin equation and thermodynamics. Progress of Theoretical Physics Supplement, 1998, 130: 17-27.

[76] JENSEN, Johan Ludwig William Valdemar, et al. Sur les fonctions convexes et les inégalités entre les valeurs moyennes. Acta mathematica, 1906, 30: $175-193$.

[77] LOH, Po-Shen. Convexity. mop2013, Carnegie Mellon University, 2013.

[78] LIPHARDT, Jan, et al. Equilibrium information from nonequilibrium measurements in an experimental test of Jarzynski's equality. Science, 2002, 296.5574: 1832-1835.

[79] COLLIN, Delphine, et al. Verification of the Crooks fluctuation theorem and recovery of RNA folding free energies. Nature, 2005, 437.7056: 231234. 